\title{
A New Type of Phase Transition Based on the Clausius-Clapeyron Relation Involving a Change in Spatial Dimension
}

\author{
Christopher Pilot \\ Physics Department, Gonzaga University, Spokane, WA, USA \\ Email: pilot@gonzaga.edu
}

How to cite this paper: Pilot, C. (2019) A New Type of Phase Transition Based on the Clausius-Clapeyron Relation Involving a Change in Spatial Dimension. Journal of High Energy Physics, Gravitation and Cosmology, 5, 291-309.

https://doi.org/10.4236/jhepgc.2019.52016

Received: January 8, 2019

Accepted: February 12, 2019

Published: February 15, 2019

Copyright ( 2019 by author(s) and Scientific Research Publishing Inc. This work is licensed under the Creative Commons Attribution International License (CC BY 4.0).

http://creativecommons.org/licenses/by/4.0/

\begin{abstract}
Using a space filled with black-body radiation, we derive a generalization for the Clausius-Clapeyron relation to account for a phase transition, which involves a change in spatial dimension. We consider phase transitions from dimension of space, $n$, to dimension of space, $(n-1)$, and vice versa, from
\end{abstract} $(n-1)$ to $n$-dimensional space. For the former we can calculate a specific release of latent heat, a decrease in entropy, and a change in volume. For the latter, we derive an expression for the absorption of heat, the increase in entropy, and the difference in volume. Total energy is conserved in this transformation process. We apply this model to black-body radiation in the early universe and find that for a transition from $n=4$ to $(n-1)=3$, there is an immense decrease in entropy accompanied by a tremendous change in volume, much like condensation. However, unlike condensation, the volume change is not three-dimensional. The volume changes from $V_{4}$, a four-dimensional construct, to $V_{3}$, a three-dimensional entity, which can be considered a subspace of $V_{4}$. As a specific example of how the equation works, we consider a transition temperature of $3 \times 10^{27} \mathrm{Kelvin}$, and assume, furthermore, that the latent heat release in three-dimensional space is $1.8 \times 10^{94}$ Joules. We find that for this transition, the internal energy densities, the entropy densities, and the volumes assume the following values (photons only). In four-dimensional space, we obtain, $u_{4}=1.15 \times 10^{125} \mathrm{~J} \cdot \mathrm{m}^{-4}, \quad s_{4}=4.81 \times 10^{97} \mathrm{~J} \cdot \mathrm{m}^{-4} \cdot \mathrm{K}^{-1}$, and $V_{4}=2.14 \times 10^{-31} \mathrm{~m}^{4}$. In three-dimensional space, we have $u_{3}=6.13 \times 10^{94} \mathrm{~J} \cdot \mathrm{m}^{-3}, s_{3}=2.72 \times 10^{67} \mathrm{~J} \cdot \mathrm{m}^{-3} \cdot \mathrm{K}^{-1}$, and $V_{3}=0.267 \mathrm{~m}^{3}$. The subscripts 3 and 4 refer to three-dimensional and four-dimensional quantities, respectively. We speculate, based on the tremendous change in volume, the explosive release of latent heat, and the magnitudes of the other quantities calculated, that this type of transition might have a connection to inflation. 
With this work, we prove that space, in and of itself, has an inherent energy content. This is so because giving up space releases latent heat, and buying space costs latent heat, which we can quantify. This is in addition to the energy contained within that space due to radiation. We can determine the specific amount of heat exchanged in transitioning between different spatial dimensions with our generalized Clausius-Clapeyron equation.

\section{Keywords}

Clausius-Clapeyron Relation, Spatial Dimension, Phase Transition, Inflation

\section{Introduction}

As is well known, the Clausius-Clapeyron relation [1] [2] [3] [4] is useful in predicting the latent heat given off when a substance undergoes a first order phase transition at a particular temperature and pressure. A first order phase transition is a discontinuous phase transition for which there is an abrupt change in phase, and latent heat is released or absorbed by a fixed amount. The discontinuity is characterized by a co-existence curve, typically plotted as pressure versus temperature, and on this curve, both phases can co-exist at specific temperatures and pressures. We assume a closed system where temperature and pressure are clearly defined on either side of this curve, and are held constant at a particular point on the curve when transitioning.

The Clausius-Clapeyron relation, as presently formulated, assumes that space is smooth, continuous, and three-dimensional, both before and after a transition. We relax the assumption of dimensionality. We will show that it is possible to generalize this important thermodynamic relation to include phase transitions, which are changing spatial dimension itself, while all the while conserving total energy. Furthermore, this kind of analysis may prove consequential in understanding the inflation phase of the early universe.

Our motivation for studying this problem is three-fold. First, it is of general theoretical interest for compactification and Kaluza-Klein theories [5]-[10]. When symmetries are broken, whether spontaneously or otherwise, the dimensionality of space often remains fixed, but not in compactification. What does it mean if spontaneous symmetry breaking occurs thermodynamically with an attendant change in spatial dimension? While we will not attempt to address this question in detail, we will show how it can be done. The key is the Clausius-Clapeyron equation.

Second, there may be possible applications to the very early universe, and specifically to inflation itself, as alluded to previously. In inflation, the universe expands exponentially and dramatically, within a very short time period, and with a rapid reduction in temperature. A discontinuous phase transition seems to offer those same characteristics except that the temperature remains fixed. A spa- 
tially changing phase transition from $n=4$ dimensions to $n=3$ dimensions, may offer the order of magnitude scales required for early cosmic evolution, and for inflation in particular. In addition, because it happened at an instant, then and there so to speak, with a tremendous release of latent heat, thermal equilibrium was guaranteed shortly thereafter. Moreover, the problem with a-causal exponential expansion may not be an issue if it is the space itself, which is expanding upon changing dimensions when transitioning. Finally, in regards to inflation, we will also show that relative fluctuations in temperature, $\delta T / T$, can be carried over, or even created in certain circumstances, when transitioning from one space to another neighboring space. This appears to be a unique feature for this kind of transformation as will be demonstrated.

Third, we recently presented a paper [11] where we advanced the notion that the universe may be modeled as a thermodynamic heat engine. There, we assumed a closed universe, i.e. one with a slight positive curvature, which will allow for a big bounce scenario. To explain inflation, and expansion in general, we proposed a Carnot cycle for the cosmos consisting of isothermal expansion (from points, $a \rightarrow b$ ), adiabatic expansion (from points, $b \rightarrow c$ ), isothermal contraction (form points, $c \rightarrow d$ ) and isothermal contraction (from points, $d \rightarrow a)$. The universe finds itself currently in the adiabatic expansion mode. This four step process brings the universe back to its initial configuration, point $a$, where we have a finite temperature, a finite pressure, a finite energy, a finite volume, etc. The universe, being cyclic, has no real beginning, nor does it have an end. Spatially, there are no "edges" as the universe has no boundaries. The inflation phase is identified as the initial isothermal expansion phase, from points, $a \rightarrow b$. This very short phase did not last long, of the order of only, $10^{-35} \mathrm{~s}$.

Time evolved very differently in the isothermal expansion mode, as was shown explicitly in reference [11]. Time evolution was not temperature dependent, but, interestingly, volume dependent. The volume expanded by a factor of only 5.65, as did total entropy, and this expansion was fueled by thermal quantum fluctuations and heat transfer from surroundings to system. We identified the "surroundings" as those parts of the observable universe, which spatially in the WMAP and Planck maps are now slightly cooler. Those are the pockets of space where matter later aggregated. The "system" consisted of voids, i.e. those parts of space that do the actual expanding currently. These regions were slightly hotter in the very early universe. The adiabatic expansion phase, which follows isothermal expansion, is driven by a different mechanism, a decrease in internal energy. The specifics are given in reference [11].

The connection between this model and a spatially changing phase transition from $n=4$ to $(n-1)=3$ space dimensions is as follows. This phase transition may have provided the impetus, quite literally, the spark, for the start of the cycle as described above. The amount of heat required for the initial isothermal expansion process, which lasted only about $10^{-35} \mathrm{~s}$, was calculated to be very high, roughly $1.8 \times 10^{94} \mathrm{~J}$. We considered only photons, and so, this estimate is, more 
than likely, on the low $\operatorname{side}^{1}$ [12] [13] [14]. We also made use of the present radius of the observable universe, about $4.4 \times 10^{26} \mathrm{~m}$, which is in itself, a very crude approximation. The temperature for the isothermal process was ascertained to be about $3 \times 10^{27} \mathrm{~K}$. This number was derived using Heisenberg's uncertainty principle, and the slight spatial temperature variations found in the WMAP and Planck missions, namely, $\delta T / T \approx \pm 5 \times 10^{-5}$ between the hot and cold spots found within the photon blackbody radiation. Perhaps the source for the heat required for the isothermal phase was not the transfer of heat from surroundings to system as originally proposed in reference [11]. Perhaps it is due to a spatially changing phase transition from $n=4$ to $(n-1)=3$ at $T \approx 3 \times 10^{27} \mathrm{~K}$. Irrespective of whether our heat engine model is valid, we consider the generalization of the Clausius-Clapeyron relation to be of paramount importance for both thermodynamics, and an understanding of compactification theory in general.

Recently, researchers [15] have suggested that a $n=4$ to $(n-1)=3$ transition might actually have occurred in the very early universe. At a temperature of $0.93 \times$ Planck Temperature they found that the Helmholtz free energy density function reaches a maximum value when plotted as a function of spatial dimension, $n=1,2,3,4, \cdots$. That maximum was reached for $n \approx 3$. This was the first of several important thermodynamic variables to do so, and they interpreted this extremum as the transition point where nature decided on three spatial dimensions. While we agree with their overall premise that compactification may have occurred, we disagree with their estimate for the temperature of this transition. The Planck temperature is $1.42 \times 10^{32} \mathrm{~K}$, and $93 \%$ of this is still $+10^{32} \mathrm{~K}$. We believe in a lower temperature for the $n=4$ to $(n-1)=3$ transition, which we call $T_{43}=T_{34}$. We believe it is closer to $3 \times 10^{27} \mathrm{~K}$ based on our heat engine ${ }^{1}$ At a temperature of $3 * 10^{27} \mathrm{~K}$, it is well known that there are many species of radiation present, not just photons. There are the neutrinos $\left(\bar{v}_{e} v_{e}, \bar{v}_{\mu} v_{\mu}, \bar{v}_{\tau} v_{\tau}\right)$, and the $e^{+} e^{-}, \mu^{+} \mu^{-}, \tau^{+} \tau^{-}$pairs which contribute to radiation. We also have quark, antiquark and gluon radiations. Then there are $W^{+}, W^{-}, Z^{0}$ radiative contributions, etc. If we take just the particles in the standard model into consideration, then we have as the energy density $u(T)=\left(\pi^{2} / 30\right) g^{*}(T) T^{4}$ where $g^{*}(T)=g_{b}(T)+7 / 8 g_{f}(T)$, and $g_{b}=\sum g_{i}$ is the sum over relativistic bosonic species. The $g_{f}=\sum g_{i}$ is the corresponding sum over relativistic fermions. The $g^{*}(T)$ counts up the effective number of relativistic degrees of freedom (photons count as two degrees of freedom), which is temperature dependent for massive particles. All particles in the standard model are already relativistic at temperatures of $10^{16} \mathrm{~K} \approx 1 \mathrm{TeV}$. When we carry out the sum for the known particle species, we obtain $g_{b}=28, g_{f}=90$, and, therefore, $g^{*}=106.75$. We would also have to add those particles which are not yet observed, but which could exist in the form of radiation at $3 * 10^{27} \mathrm{~K}$ such as supersymmetric particles, dark matter particles, etc. To make a long story short, the input heat needed to bring these types of radiations into thermal equilibrium with the photons is therefore higher, than if we just consider photons by themselves. Therefore, our original rough estimate of $L=1.8 * 10^{94} \mathrm{~J}$ is, most probably, too low in value and we should multiply this number by a scale factor such as $g^{*}(T)$ in order to take into account other species of radiations. We focus only on the photon contribution to keep the discussion simple, but also because we cannot be sure as to what contributes at this extremely high temperature. In addition, we have to remember that our estimate for latent heat is, in itself, a very rough approximation to begin with. Two good references on relativistic degrees of freedom and their contribution to radiation, are references [13] and [14], which follow. 
model, as well as other considerations. Regardless of what the actual transition temperature turns out to be, assuming it exists, we approach the problem of a spatial phase transition from an entirely different perspective. We focus on the Clausius-Clapeyron (abbreviated CC) relation and generalize the relation to apply for a spatial change in phase; in other words, the dimension of space changes.

The outline of this paper is as follows. In Section II, we generalize the CC relation using radiation as the substance filling space. We believe that radiation in all its forms (photons, neutrinos, $\mathrm{e}^{+} \mathrm{e}^{-}$pairs, etc.) is the primordial substance found in the very early universe when temperatures were very high. Radiation will define space according to Mach's principle (matter/energy content defines space) and spatial transitions are assumed possible. To keep the discussion simple we will focus exclusively on photons. In very general terms we derive the generalization of the CC equation for an arbitrary $n$-dimensional to $(n-1)$-dimensional spatial change of phase, and vice versa. We also consider the conservation of energy and changes in hypervolume in general terms. In Section III we focus on the transition from $n=4$ to $(n-1)=3$. We will assume specific values for temperature of transition, as well as amount of latent heat release, in order to show how the equation works. The specific values chosen are motivated by previous work [11]. Quantities in three-dimensional and four-dimensional spaces are then calculated, such as entropy and volume, both before and after. In Section IV, we discuss inflation in general, and consider our $n=4 \rightarrow(n-1)=3$ model in particular. The WMAP and Planck satellite missions show a remarkable uniformity in photon blackbody temperature. Nevertheless, there is a slight inhomogeneity in temperature, which explains the present structure of the universe. That inhomogeneity is of the order, $\delta T / T= \pm 5 \times 10^{-5}$. How does this non-uniformity in temperature behave when undergoing a spatially changing phase transition? How, specifically, are the other thermodynamic quantities affected? We will answer both questions in Section IV. Finally, in Section V, we present our summary and conclusions.

\section{Generalization of the Clausius-Clapeyron Relation}

In this section, we generalize the CC relation to allow for a phase transition from $n$-dimensional space to $(n-1)$-dimensional space and vice versa. We start with the internal internal radiation energy density (photons only). As is known [16] [17] [18] [19], the internal energy density in $n$-dimensional space is given by the following function, which depends only on temperature and the dimensionality of space, $n$ :

$$
u=u(n, T)=2(n-1) \pi^{n / 2}\left(k_{B} T\right)^{n+1} \zeta(n+1) \Gamma(n+1) /\left[(h c)^{n} \Gamma(n / 2)\right]
$$

In this equation, $k_{B}$ is Boltzmann's constant, $c$ equals the speed of light, $h$ is Planck's constant, $\zeta(x)$ is the zeta function, and $\Gamma(x)$ is the gamma function. From this function, we can furthermore show that

$$
f=-u / n, \quad p=u / n, \quad s=(n+1) / n u / T
$$


Here, " $P$ " is the Helmholtz free energy density, " $p$ " is the pressure, and " $s$ " is the entropy density. The Helmholtz function is defined as $F \equiv U-T S$, and therefore, $f=u-T s$.

In $n$-dimensional space, a hypervolume can be defined for a $n$-dimensional ball. The expression [20] [21] is

$$
V_{n}=V_{n}\left(R_{n}\right)=\pi^{\frac{n}{2}}\left(R_{n}\right)^{n} / \Gamma\left(\frac{n}{2}+1\right)
$$

The subscript " $n$ " on a physical quantity will always refer to the spatial dimension in which the quantity is defined. $\Gamma(x)$ is again the gamma function.

If we specialize to three spatial dimensions, $n=3$, then we obtain familiar formulas using the equations above:

$$
u_{3}=8 / 15 \pi^{5}\left(k_{B} T\right)^{4} /(h c)^{3}, p_{3}=u_{3} / 3, s_{3}=4 / 3 u_{3} / T, V_{3}=4 / 3 \pi R_{3}^{3}
$$

The internal energy density is often written as $u_{3}=4 \sigma T^{4} / c=A T^{4}$, where $\sigma$ is the Stefan-Boltzmann constant, and $A$ has the numerical value equal to $7.566 \times 10^{-16} \mathrm{~J} \cdot \mathrm{m}^{-3} \cdot \mathrm{K}^{-4}$. For $=4, V_{4}$ equals $\left(\pi^{2} / 2\right)\left(R_{4}\right)^{4}$, and in two dimensions, $V_{2}=\pi\left(R_{2}\right)^{2}$. When not specified explicitly, we use MKS units throughout this paper.

Next, we consider the entropy in $n$-dimensional space. We find that

$$
S_{n}=S_{n} V_{n}=(n+1) / n\left(u_{n} / T\right) V_{n}
$$

For $(n-1)$-dimensional space, we obtain

$$
S_{n-1}=S_{n-1} V_{n-1}=n /(n-1)\left(u_{n-1} / T\right) V_{n-1}
$$

We can also calculate, using Equation (2-2) and Equation (2-1), $\left(\mathrm{d} p_{n} / \mathrm{d} T\right) V_{n}$. The result is

$$
\left(\mathrm{d} p_{n} / \mathrm{d} T\right) V_{n}=(n+1) / n\left(u_{n} / T\right) V_{n}
$$

Similarly,

$$
\left(\mathrm{d} p_{n-1} / \mathrm{d} T\right) V_{n-1}=n /(n-1)\left(u_{n-1} / T\right) V_{n-1}
$$

Comparing right hand sides of Equation (2-5) and Equation (2-7), it is clear that

$$
S_{n}=\left(\mathrm{d} p_{n} / \mathrm{d} T\right) V_{n}
$$

Similarly, comparing right hand sides of (2-6) and (2-8), we see that

$$
S_{n-1}=\left(\mathrm{d} p_{n-1} / \mathrm{d} T\right) V_{n-1}
$$

Therefore, if we take the difference between Equation (2-9) and Equation (2-10), we find that

$$
S_{n}-S_{n-1}=\left(\mathrm{d} p_{n} / \mathrm{d} T\right) V_{n}-\left(\mathrm{d} p_{n-1} / \mathrm{d} T\right) V_{n-1}
$$

This is our generalization of the CC relation. The difference in entropy multiplied by the temperature is the latent heat, $\Delta Q$. Therefore, Equation (2-11) can also be written as 


$$
S_{n}-S_{n-1}=\left(\mathrm{d} p_{n} / \mathrm{d} T\right) V_{n}-\left(\mathrm{d} p_{n-1} / \mathrm{d} T\right) V_{n-1}=1 / 2 \Delta Q / T
$$

The factor of $1 / 2$ on the right hand side of Equation (2-12) will be explained shortly. Equation (2-12) is dimensionally consistent, as we shall also soon see, even though the densities and pressure are defined in different dimensions, and thus have different units.

The general expression for $\Delta S=\left(S_{f}-S_{i}\right)$ is $\Delta S=\int_{i}^{f} \mathrm{~d} Q / T$. The $S_{n}$ and $S_{n-1}$ in Equation (2-12) can be thought of as entropy states, $S_{i}$ and $S_{f}$. However, if the temperature is held fixed, as in a first order phase transition, this reduces to $\Delta S=\Delta Q / T$. When written out, $\left(S_{f}-S_{i}\right)=\left(Q_{f}-Q_{i}\right) / T$. The sign of $\Delta S$ determines the sign of $\Delta Q$. It will soon become apparent that $S_{n}=S_{i}$ is always greater than $S_{n-1}=S_{f}$. Therefore, $\Delta Q$ in (2-12) is positive which means that heat is being given off in the final state. If we reverse the transition from $(n-1)$-space to $n$-space, we simply multiply Equation (2-12) by a minus sign. In this instance, $S_{n}=S_{f}$ and $S_{n-1}=S_{i}$ and $\Delta Q$ is negative. This means that latent heat has to be supplied for this transition to occur. The $\Delta Q$ is often written as $L$, which stands for latent heat. Barring exotic scenarios where we have parallel universes or multi-universes, etc., we will assume that the latent heat, which is released in the first type of transition where we decrease the number of dimensions, will be released in $(n-1)$-space. For the second type of transition, where we increase the dimensionality of space, the heat which needs to be supplied in order for this transition to happen, needs to come from the originating $(n-1)$-space.

Equation (2-12) reduces to the conventional CC relation (up to a factor of 1/2) in the limit where $n$ equals $(n-1)$, if we can imagine such a limit allowing for $S_{n} \neq S_{n-1}$ and $V_{n} \neq V_{n-1}$. Both temperature and dimension of space are similar in this limit, and thus, there is no difference between $p_{n}$ and $p_{n-1}$. We retrieve the standard CC equation in Equation (2-12), except for the factor of $1 / 2$. Therefore, in an intriguing way, the familiar CC relation is obtained as a special case when neighboring spaces converge. Since a first order phase transition is a discontinuous phase transition, we can easily imagine that $S_{n} \neq S_{n-1}$ and $V_{n} \neq V_{n-1}$, even though the dimensions of space are now the same in this special limit.

Let us next prove that Equation (2-12) is dimensionally consistent. We note that, in terms of units, the $\operatorname{dim}\left[T_{n}\right]=\operatorname{dim}\left[T_{n-1}\right]=\operatorname{dim}[T]$. However, Equation (2-1) shows us that

$$
\operatorname{dim}\left[u_{n}\right]=\mathrm{J} \cdot \mathrm{m}^{-n} \neq \operatorname{dim}\left[u_{n-1}\right]=\mathrm{J} \cdot \mathrm{m}^{-(n-1)}
$$

We are working within the MKS system where "J" stands for Joules and " $m$ " for meters. From relations (2-13) and (2-2b), we also notice that

$$
\operatorname{dim}\left[p_{n}\right]=\operatorname{dim}\left[u_{n}\right]=\mathrm{N} \cdot \mathrm{m}^{-(n-1)} \neq \operatorname{dim}\left[p_{n-1}\right]=\operatorname{dim}\left[u_{(n-1)}\right]=\mathrm{N} \cdot \mathrm{m}^{-(n-2)}
$$

Here " $\mathrm{N}$ " refers to Newtons $=\mathrm{J} \cdot \mathrm{m}^{-1}$. Furthermore, upon using Equation (2-2c), we find 


$$
\operatorname{dim}\left[s_{n}\right]=\mathrm{J} \cdot \mathrm{m}^{-n} \cdot \mathrm{K}^{-1} \neq \operatorname{dim}\left[s_{n-1}\right]=\mathrm{J} \cdot \mathrm{m}^{-(n-1)} \cdot \mathrm{K}^{-1}
$$

The "K" refers to degrees Kelvin. Moreover, from Equation (2-3) we see that

$$
\operatorname{dim}\left[V_{n}\right]=m^{n} \neq \operatorname{dim}\left[V_{(n-1)}\right]=m^{(n-1)}
$$

From these relations, it is easy to prove that

$$
\begin{aligned}
& \operatorname{dim}\left[U_{n}\right]=\operatorname{dim}\left[u_{n}\right] * \operatorname{dim}\left[V_{n}\right]=\mathrm{J}=\operatorname{dim}\left[U_{n-1}\right]=\operatorname{dim}\left[u_{n-1}\right] * \operatorname{dim}\left[V_{n-1}\right] \\
& \operatorname{dim}\left[S_{n}\right]=\operatorname{dim}\left[s_{n}\right] * \operatorname{dim}\left[V_{n}\right]=\mathrm{J} / \mathrm{K}=\operatorname{dim}\left[S_{n-1}\right]=\operatorname{dim}\left[s_{n-1}\right] * \operatorname{dim}\left[V_{n-1}\right]
\end{aligned}
$$

The quantities, $U_{n}$ and $S_{n}$, refer to the internal energy and entropy in $n$-spatial dimensions, and we notice that these quantities do not depend on the value of " $n$ " as far as dimensional units are concerned. We can substitute the dimensionalities specified above into Equation (2-12) to show that the Equation $(2-12)$ is, indeed, dimensionally correct.

We now explain the factor of 1/2 in Equation (2-12). Conservation of energy, in all its forms, between spatial dimensions demands that

$$
U_{n}+p_{n} V_{n}+S_{n} T=U_{n-1}+p_{n-1} V_{n-1}+S_{n-1} T+L
$$

In this equation, $L$ is the latent heat released in $(n-1)$-dimensional space, which may or may not equal zero, at this stage (It will turn out that $L$ is unequal to zero and positive later). The various terms on the left hand side of (2-19) represent the internal energy, the stored work, and the heat content of photons, respectively, in $n$-dimensional space. We have the same on the right hand side but in $(n-1)$-dimensional space, plus any latent heat, which may, or may not, be released in $(n-1)$ space. We can simplify Equation (2-19), utilizing Equation (2-2b). Upon substitution of the latter expression, we now write (2-19) as

$$
[(n+1) / n] U_{n}+S_{n} T=[n /(n-1)] U_{n-1}+S_{n-1} T+L
$$

We can simplify (2-19) further using (2-2c) to eliminate $S_{n}$ and $S_{n-1}$. Here we obtain

$$
2[(n+1) / n] U_{n}=2[n /(n-1)] U_{n-1}+L
$$

Alternatively, we use Equation (2-2c) to eliminate $U_{n}$ and $U_{n-1}$ in Equation (2-20) and find that

$$
2 S_{n} T=2 S_{n-1} T+L
$$

However, from the paragraph following Equation (2-12), we saw that

$$
S_{f}-S_{i}=S_{n-1}-S_{n}=\left(Q_{f}-Q_{i}\right) / T=\left(Q_{n-1}-Q_{n}\right) / T=-L / T
$$

The subscripts, $i$ and $f$, stand for initial and final states, and $L$ refers to the latent heat, which will be a positive quantity. Upon comparison of the two expressions, Equation (2-22) and Equation (2-23), we notice that Equation (2-23) is really missing a factor of $1 / 2$. When transitioning between different dimensions, photons need to maintain their identity in each spatial dimension, the initial dimension and the final dimension. This leads to additional terms involving internal energy and stored work in Equation (2-19), on both left and right hand 
sides. As it turns out, the sum of internal energy and stored work is numerically equal, in each dimension, to the stored heat in that dimension. Therefore, we have the extra factor of two in both Equation (2-21) and Equation (2-22). Wherever we see $Q$ or $L$ in Equation (2-23), we should substitute $1 / 2 Q$, and $1 / 2 L$. Another way of saying the same thing is that twice the entropy change is needed to release a fixed amount of latent heat, $L$, due to the requirement of maintaining internal energy and stored work, in both spaces. See Equation (2-22). Equation $(2-22)$ is another way to write our generalized CC equation, Equation (2-12). One cannot just transfer internal energy for photons, and leave the associated pressure and entropy behind. It's all or nothing if a transition occurs.

We close this section by deriving an expression for the hypervolume ratio, $\left(V_{n} / V_{n-1}\right)$, as this will be needed later on. We start with Equation (2-21), which we rewrite as

$$
2[(n+1) / n] u_{n} V_{n}=2[n /(n-1)] u_{n-1} V_{n-1}+L_{n-1}
$$

On the right hand side of (2-24), we have made explicit the fact that $L$ is defined in $(n-1)$-space. We next define latent heat density as $l_{n} \equiv L_{n} / V_{n}$. This allows us to reformulate Equation (2-24) as follows:

$$
V_{n} / V_{n-1}=\left[n^{2} /\left(n^{2}-1\right)\right] u_{n-1} / u_{n}+[n / 2(n+1)] l_{n-1} / u_{n}
$$

Therefore,

$$
V_{n} / V_{n-1}=u_{n-1} / u_{n}\left[n^{2} /\left(n^{2}-1\right)+n / 2(n+1) l_{n-1} / u_{n-1}\right]
$$

or, alternatively,

$$
V_{n} / V_{n-1}=u_{n-1} / u_{n}\left[n^{2} /\left(n^{2}-1\right)+n / 2(n+1) L_{n-1} / U_{n-1}\right]
$$

The latent heat is released in $(n-1)$ space for a transition from spatial dimension, $n$, to spatial dimension, $(n-1)$. As mentioned previously, we will not consider exotic situations where the heat can be released in any other kind of space, such as in a parallel universe, multi-universes, etc.

Both Equation (2-26) and Equation (2-27), are linear equations where the dependent variable can be considered $\left(V_{n} / V_{n-1}\right)$ and the independent variable is either $l_{n-1}$ for Equation (2-26), or $L_{n-1}$ for Equation (2-27). We will be looking at a transition from $n=4$ to $(n-1)=3$ in the next section, and it is more likely that we can give an estimation of either $l_{n-1}$ or $L_{n-1}$, versus $\left(V_{n} / V_{n-1}\right)$. Hence, we treat the latter as the dependent variable. Given a specific transition temperature, all other quantities can be determined or estimated on the right hand sides of Equation (2-26) and Equation (2-27). We can make use of the general expression, Equation (2-1), to determine $u_{n}$ and $u_{n-1}$ for a specific transition temperature. For $U_{3}$ we need to give the size of the observable universe, $V_{3}$. However, we can estimate this volume for a particular transition temperature. The present size [22] [23] of the observable universe is approximately $4.4 \times 10^{26} \mathrm{~m}$ in radius, and this radius is scaled down by the cosmic scale factor, $a=T_{0} / T$, for any other temperature $T$. We will ignore slight kinks due to 
$\mathrm{e}^{+} \mathrm{e}^{-}$radiation annihilation and heating up of photons. For $T$ we substitute $T_{43}$, the transition temperature, and for $T_{0}$, we insert the present temperature of the photon blackbody radiation, which is $T_{0}=2.7255 \mathrm{~K}$. Therefore, we estimate that $V_{3}=a^{3} \times($ present observable volume $)=a^{3} V_{0}=a^{3}(4 \pi / 3)\left(4.4 \times 10^{26} \mathrm{~m}\right)^{3}$. For a specific transition temperature of $3 \times 10^{27} \mathrm{~K}$, we obtain $V_{3}=0.267 \mathrm{~m}^{3}$.

We can easily read off the slope and y-intercept, in both Equation (2-26) and Equation (2-27). Both slope and y intercept are transition temperature dependent, and only transition temperature dependent for a given " $n$ " to $(n-1)$ transition. For any latent heat release in $(n-1)$ space, we can calculate the volume in $n$-space using either Equation (2-26) or Equation (2-27).

\section{The $n=4$, to $(n-1)=3$, Transition}

In this section, we consider the $n=4$ to $(n-1)=3$ transition. We start with the generalized CC equation, Equation (2-12). We specialize to $n=4$, and obtain

$$
\left(S_{4}-S_{3}\right)=\left(\mathrm{d} p_{4} / \mathrm{d} T\right) V_{4}-\left(\mathrm{d} p_{3} / \mathrm{d} T\right) V_{3}=1 / 2 \Delta Q / T
$$

Written more elegantly, we use Equation (2-22), which is the equivalent. We focus on this second version, and write

$$
\left(S_{4}-S_{3}\right)=1 / 2 L / T
$$

Our task is to find the hypervolume, $V_{4}$, using this equation, as well as other thermodynamic quantities of interest in 3-space and 4-space for a specific transition temperature. We start with Equation (2-1), where we first evaluate $u_{3}$ and $u_{4}$. We will assume a transition temperature of $3 \times 10^{27} \mathrm{~K}$. Upon evaluating the constants and inserting this temperature, we find:

$$
\begin{aligned}
& u_{3}=A T^{4}=7.566 \times 10^{-16} \times T^{4}=6.128 \times 10^{94} \mathrm{~J} / \mathrm{m}^{3} \\
& \text { and } \\
& \begin{aligned}
u_{4} & =u_{3} \times \zeta(5) / \zeta(4) \times\left(k_{B} T\right) /(\hbar c) \times 3 / 2=u_{3} \times 1.437 \times\left(k_{B} T\right) /(\hbar c) \\
& =u_{3} \times 627.6 \times T=1.154 \times 10^{125} \mathrm{~J} / \mathrm{m}^{4}
\end{aligned}
\end{aligned}
$$

MKS units will be used exclusively in this paper (even though, sometimes, we will not always write them out). We next evaluate the radiation pressure in both spaces. Using (2-2b) and Equation (3-3), we obtain

$$
p_{3}=u_{3} / 3=2.043 \times 10^{94} \mathrm{~N} / \mathrm{m}^{2} \text { and } p_{4}=u_{4} / 4=2.884 \times 10^{124} \mathrm{~N} / \mathrm{m}^{3}
$$

For the entropy density we utilize (2-2c) and Equation (3-3), and discover that $s_{3}=4 u_{4} / 3 T=2.724 \times 10^{67} \mathrm{~J} /\left(\mathrm{m}^{3} \cdot \mathrm{K}\right)$ and $s_{4}=5 u_{4} / 4 T=4.807 \times 10^{97} \mathrm{~J} /\left(\mathrm{m}^{4} \cdot \mathrm{K}\right)$

Furthermore, we know the value of $V_{3}$. This was evaluated in the last section, in the paragraph following Equation (2-27). The result for a transition temperature of $3 \times 10^{27} \mathrm{~K}$ was $V_{3}=0.267 \mathrm{~m}^{3}$. With this result, we can evaluate both $U_{3}$ and $S_{3}$ explicitly. The results are

$$
U_{3}=u_{3} V_{3}=1.639 \times 10^{94} \mathrm{~J} \text { and } S_{3}=S_{3} V_{3}=7.283 \times 10^{66} \mathrm{~J} / \mathrm{K}
$$

We have made use of Equation (3-3a) and Equation (3-5a). 
We next calculate $S_{4}$. For this, we have to assume a value for the latent heat. We adopt as a value, $L=1.8 \times 10^{94} \mathrm{~J}$, a number which was motivated to some extent in the introduction. Using this value in Equation (3-2) renders

$$
S_{4}-S_{3}=3.000 \times 10^{66} \mathrm{~J} / \mathrm{K}
$$

In addition, from Equation (3-6), we have a value for $S_{3}$. Inserting this into Equation (3-7), we find that $S_{4}$ equals $1.028 \times 10^{67} \mathrm{~J} / \mathrm{K}$. Finally, we have a value for $s_{4}$, as this was numerically evaluated in Equation (3-5b). We can therefore obtain the hypervolume, $V_{4}$, by taking $S_{4}$ and dividing out by $S_{4}$. The result is

$$
V_{4}=S_{4} / s_{4}=1.028 \times 10^{67} / 4.807 \times 10^{97}=2.139 \times 10^{-31} \mathrm{~m}^{4}
$$

This is a fantastically small volume. To obtain a $3-\mathrm{d}$ volume, $V_{3}=0.267 \mathrm{~m}^{3}$, from a volume such as this, a dimension of space must have curled up on itself to compactify to $V_{3}$. If we call that dimension, which has compactified, the $W$-dimension, then we notice that

$$
w=V_{4} / V_{3}=2.139 \times 10^{-31} / 0.267=7.999 \times 10^{-31} \mathrm{~m} .
$$

Now that we have $V_{4}$, we can find the internal energy, $U_{4} \cdot U_{4}$ is obtained by multiplying the internal energy density in 4 -d space, $u_{4}$, by the hypervolume, $V_{4}$. Using the results of Equation (3-3b) and Equation (3-8), we find

$$
U_{4}=u_{4} V_{4}=2.468 \times 10^{94} \mathrm{~J}
$$

We check our results by verifying our energy balance equation, Equation (2-19). Equation (2-19) reads for $n=4$ :

$$
U_{4}+p_{4} V_{4}+S_{4} T=U_{3}+p_{3} V_{3}+S_{3} T+L
$$

Upon substitution of Equations (3-9), (3-4b), (3-8), (3-7) with (3-6b), (3-6a), (3-4a) with $V_{3}=0.267 \mathrm{~m}^{3},(3-6 \mathrm{~b})$ and $L=1.8 \times 10^{94} \mathrm{~J}$, we have, term for term,

$$
\begin{gathered}
2.468 \times 10^{94}+0.617 \times 10^{94}+3.085 \times 10^{94} \\
=1.639 \times 10^{94}+0.546 \times 10^{94}+2.185 \times 10^{94}+1.8 \times 10^{94} \\
6.17 \times 10^{94} \mathrm{~J}=6.17 \times 10^{94} \mathrm{~J}
\end{gathered}
$$

Our energy equation balances, and it is clear that $L$ is a positive quantity as claimed previously. Furthermore, we notice that in 4 -d space, as well as in 3 -d space, the sum, $\left(U_{n}+p_{n} V_{n}\right)$, always equals $S_{n} T$. This is apparent in Equation (3-10), on both left and right hand sides, when evaluating a sum of the first two terms and comparing with the third term.

We could have obtained the hypervolume, $V_{4}$, more directly using Equation (2-27). However, then, we would not have had the opportunity to specify the other thermodynamic variables. Specializing Equation (2-17) for a $n=4$ to $(n-1)=3$ transition, we obtain

$$
V_{4} / V_{3}=u_{3} / u_{4}\left[16 / 15+2 / 5 \times L / U_{3}\right]
$$

The ratio, $u_{4} / u_{3}$ equals $(627.6 \times T)$ from Equation $(3-3 \mathrm{~b})$. Assuming a transition temperature of $3 \times 10^{27} \mathrm{~K}$, this gives $u_{4} / u_{3}=1.883 \times 10^{30} . U_{3}$ is specified in Equation (3-6a). Moreover, $L$ is assumed to equal $1.8 \times 10^{94} \mathrm{~J}$. The volume, 
$V_{3}$, was determined from the transition temperature and has a value of $0.267 \mathrm{~m}^{3}$. Substituting all this into Equation (3-11) gives the result obtained earlier, namely that $V_{4}=2.139 \times 10^{-31} \mathrm{~m}^{4}$, which is Equation (3-8).

If we do not assume a particular value for the latent heat, then Equation (3-11) is a linear equation where we treat $V_{4} / V_{3}$ as the dependent variable, and $L$ is the independent variable. The y-intercept is $(16 / 15)\left(u_{3} / u_{4}\right)$, which is a constant at a specified transition temperature. The slope equals

$$
(2 / 5)\left(u_{3} / u_{4}\right) / U_{3}=(2 / 5) /\left(u_{4} V_{3}\right) \text {. }
$$

This is also a constant for a specified transition temperature because of Equation (2-1) and since $V_{3}=a^{3} V_{0}=a^{3}(4 \pi / 3)\left(4.4 \times 10^{26} \mathrm{~m}\right)^{3}$ where $a=T_{0} / T_{43}$. To be specific, we will assume a transition temperature of $T_{43}=T_{34}=3 \times 10^{27} \mathrm{~K}$. We evaluate the quantities on the right hand side of Equation (3-11), but keep the latent heat value, $L$, open. Our specific expression for this transition temperature becomes

$$
V_{4} / V_{3}=1.296 \times 10^{-125} \times L+5.666 \times 10^{-31}
$$

A plot of $V_{4} / V_{3}$ versus $L$ is illustrated in Figure 1, for various $L$ values. The linearity is apparent. For $L=0$, we obtain $V_{4} / V_{3}=5.666 \times 10^{-31} \mathrm{~m}$. Moreover, if $L$ assumes a very large value, such as $1 \times 10^{100}$ Joules, then we find correspondingly, that $V_{4} / V_{3}=1.297 \times 10^{-25} \mathrm{~m}$. Utilizing Equation (3-12), we can assume any value for latent heat and find the corresponding ratio of volumes.

\section{Inflation as a $n=4$ to $n=3$ Phase Transition}

Inflation is needed in order to explain the relative homogeneity in temperature found in the very early universe, as well as the slight inhomogeneity. The universe underwent a phase transition where there was rapid a-causal exponential expansion of the universe. The theory invokes a scalar field, the inflaton field, which drives this expansion. In the introduction, we discussed a heat engine model for the universe, where inflation is treated somewhat differently. It was identified with an initial isothermal expansion phase, where the expansion was

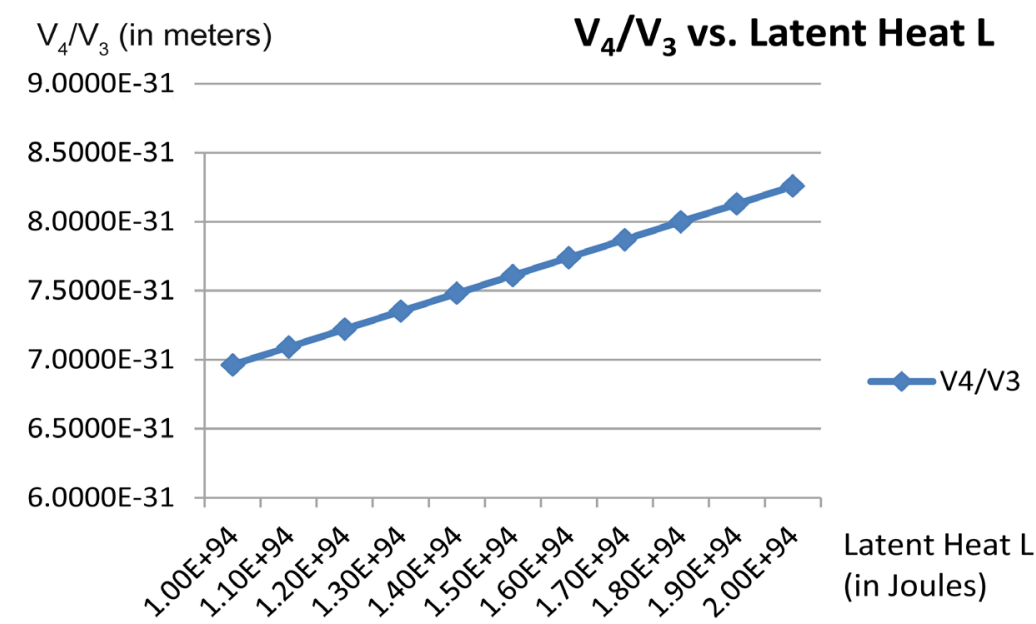

Figure 1. $V_{4} / V_{3}$ vs. Latent Heat $L$. 
not as drastic, where there was no inflaton field, and where heat input from surroundings to system drove the process. In this model for inflation, the 3-d volume increased by a factor of only 5.65. In this paper, we entertain the notion that the heat input needed is produced by a spatially changing phase transition. This is an alternative model, or perhaps complementary model, to heat input flowing from surroundings to system. We speculate that inflation is still an isothermal transition, but what provides the impetus for initiation of the heat cycle is a $n=4$ to $(n-1)=3$ change in space dimension. There is a substantial amount of heat released in such a transition as was demonstrated in the previous section. The energy densities and entropy were also significant. This may be an alternative or complementary source of heat to drive the inflation process, in our view.

The inhomogeneity in temperature found in the WMAP and Planck satellite data, of the order of $\delta T / T= \pm 5 \times 10^{-5}$, is thought to have produced during this inflationary period. These thermal fluctuations were due to quantum mechanical effects, radiative corrections induced by virtual particle creation and annihilation. The point is that they were heat driven, and since our thermodynamic variables depend critically on temperature, a natural question to ask is how do the thermodynamic quantities, introduced previously, depend on these thermal perturbations? Moreover, what happens to these fluctuations if a spatially changing phase transition takes place? These are the questions, which we will address in this section.

Quite generally, given the fact that the thermodynamic variables for radiation depend strictly on temperature and dimension of space, we can vary each thermodynamic quantity with respect to temperature. We start with the internal energy density, Equation (2-1). Varying this with respect to temperature, we find that

$$
\delta u_{n} / u_{n}=(n+1) \delta T / T
$$

Similarly, using Equation (2-2), we can further claim that

$$
\delta f_{n} / f_{n}=(n+1) \delta T / T, \delta p_{n} / p_{n}=(n+1) \delta T / T, \delta s_{n} / s_{n}=n \delta T / T
$$

From these relations, we see that the dimensionality of space plays a role in determining how the thermodynamic entity responds to a relative fluctuation in temperature. In addition, quite generally, we will assert that, if the process is adiabatic in $n$-space, then

$$
\delta V_{n} / V_{n}=-n \delta T / T
$$

We will be assuming that a change in cosmic scale parameter in any dimension " $n$ " is inversely proportional to temperature. Just as $a=R / R_{0}=T_{0} / T$ holds in 3-d space, we are claiming that in $n$-dimensional space,

$$
a_{n}=R_{n} / R_{0 n}=T_{0} / T
$$

provided we have adiabatic expansion in that $n$-space. In Equation (4-4), $R_{n}$ is the radius of the hypervolume in $n$-dimensional space and $R_{n 0}$ is some base- 
line radius in that same space. $R_{n 0}$ corresponds to $T_{0}$ whereas $R_{n}$ corresponds to $T$. The " $a_{n}$ " is chosen such that, at temperature $T=T_{0}$, we have $a_{0 n}=1$.

To prove Equation (4-3), we notice that Equation (2-3) allows us to express the hypervolume as $V_{n}=C R_{n}^{n}$ where $C$ is some constant of order unity. Therefore, $\delta V_{n}=n C R_{n}^{n-1} \delta R_{n}$ and $\delta V_{n} / V_{n}=n \delta R_{n} / R_{n}$. Next, we utilize Equation (4-4), which holds only for adiabatic expansion, and write $\delta R_{n} / R_{n}=-\delta T / T$. Substituting this into our expression for $\delta V_{n} / V_{n}$ gives $\delta V_{n} / V_{n}=-n \delta T / T$, which is our Equation (4-3).

With Equation (4-3), we can demonstrate that

$$
\begin{aligned}
\delta U_{n} & =\delta u_{n} V_{n}+u_{n} \delta V_{n} \\
& =(n+1) u_{n} V_{n} \delta T / T-n u_{n} V_{n} \delta T / T \\
& =U_{n} \delta T / T
\end{aligned}
$$

Therefore, $\delta U_{n} / U_{n}=\delta T / T$. Similarly, we find for any value of " $n$ ",

$$
\delta\left(p_{n} V_{n}\right) /\left(p_{n} V_{n}\right)=\delta T / T, \quad \delta S_{n} / S_{n}=0
$$

We also recognize from Equation (2-22), and Equation (4-6b), that $\delta(L / T)$ must equal zero. Therefore, it follows that

$$
\delta L / L=\delta T / T
$$

This equation tells us that temperature fluctuations produce proportional latent heat fluctuations within a specified region of space. The relations, Equations (4-5)-(4-7), do not depend explicitly on spatial dimension. They do assume adiabatic expansion on both sides of the transition curve.

The conservation of energy, Equation (2-19), can be written in the simplified form, Equation (2-21). Employing Equation (4-5) and Equation (4-7), it is obvious that from Equation (2-21),

$$
\begin{gathered}
2[(n+1) / n] \delta U_{n}=2[n /(n-1)] \delta U_{n-1}+\delta L \\
2[(n+1) / n] U_{n} \delta T / T=\left\{2[n /(n-1)] U_{n-1}+L\right\} \delta T / T
\end{gathered}
$$

This equation shows that for adiabatic expansion or contraction between two neighboring spaces, any spatial temperature fluctuations carry through undiminished from one space to the next. Therefore, if we consider a $n=4$ to $(n-1)=3$ transition, a spatial fluctuation in temperature in $(n-1)=3$ space transfers over into $n=4$ space. Equation (4-3) was critical in establishing Equation (4-8). Moreover, Equation (4-3) depended in turn on relation (4-4).

What happens, however, if, in $n$-dimensional space and in its neighboring $(n-1)$ space, we do not have adiabatic expansion or contraction? For example, in the heat engine model described in the introduction, isothermal expansion preceded adiabatic expansion. At point " $a$ " in the Carnot cycle, isothermal expansion started. At point " $b$ ", isothermal expansion changed to adiabatic expansion. Between points " $a$ " and " $b$ ", the expansion is strictly isothermal, and between points " $b$ " and " $c$ ", it is strictly adiabatic. If a spatial transition occurred 
anywhere within that time, then we cannot assume that Equation (4-4) holds. In this instance, we claim that thermal fluctuations could have been created or produced within the transition itself.

To demonstrate this, let us assume a $n=4$ to $(n-1)=3$ transition. We specialize Equation (2-21) to this situation, and vary that equation. We find that

$$
10 / 4 \delta U_{4}=8 / 3 \delta U_{3}+\delta L
$$

We divide the left hand side of this equation by the left hand side of Equation (2-21) and we do the same on the right hand side. In this way we obtain after some algebraic manipulation

$$
\begin{aligned}
\delta U_{4} / U_{4} & =\left(8 / 3 \delta U_{3}+\delta L\right) /\left(8 / 3 U_{3}+L\right) \\
& =\left(\delta U_{3} / U_{3}+3 / 8 \delta L / U_{3}\right) /\left(1+3 / 8 L / U_{3}\right)
\end{aligned}
$$

For $\delta U_{3} / U_{3}$, we substitute $\delta T / T$ because of Equation (4-5). We are assuming that in 3-d space, after point " $b$ " in the cycle, we do have adiabatic expansion. This gives

$$
\delta U_{4} / U_{4}=\left(\delta U_{3} / U_{3}+3 / 8 \delta L / U_{3}\right) /\left(1+3 / 8 L / U_{3}\right)
$$

Furthermore, let us assume that $\delta U_{4} / U_{4}=0$. This would mean a perfectly smooth spatial internal energy distribution, as well as total energy distribution in the originating 4-space, with absolutely no temperature perturbations. With this assumption, both the left and the right hand sides of Equation (4-11) equal zero, and we're left with

$$
\delta T / T=-3 / 8 \delta L / U_{3}
$$

Finally, we substitute some numerical values for the quantities in Equation (4-12). For $\delta T / T$, we take $\pm 5 \times 10^{-5}$, and for $U_{3}$ let us use the value indicated by (3-6a). In Equation (4-12), these values give

$$
\delta L=-/+2.185 \times 10^{90} \text { Joules }
$$

The $\delta L$ is defined in 3-d space and it is a small thermal perturbation when compared with $L=1.8 \times 10^{94} \mathrm{~J}$. See Equation (3-10). From Equation (4-12), it is clear that an increase in temperature for the photons in a spatial pocket leads to a decrease in latent heat in that region. The converse holds, i.e. a decrease in temperature for photons spatially will produce an increase in latent heat in that particular region of space. This is opposite to what we had previously, for neighboring spaces where adiabatic expansion/contraction holds in each space on either side of the transition curve.

By means of this simple example, we have shown that spatial temperature fluctuations can be literally produced or created in a neighboring space even though none existed in the originating space. We cannot assume adiabatic expansion or contraction in both spaces though. At least one space has to be different in this regard. This result can be extended to any $n$-space. It would appear that this is a necessary condition for creation of temperature inhomogeneity when transitioning between different spaces. 


\section{Summary and Conclusions}

We have generalized the Clausius-Clapeyron (CC) relation to take into account a type of phase transition for which there is a change in spatial dimension. In going from $n$-dimensional space to $(n-1)$-dimensional space we have a release of latent heat, a decrease in entropy, a decrease in energy density, and a change in volume from $V_{n}$ to $V_{n-1}$. In transitioning from $(n-1)$ dimensions in space to $n$ dimensions, latent heat is absorbed, with an accompanying increase in entropy, energy density, and a change in volume from $V_{n-1}$ to $V_{n}$. The generalization can be written as Equation (2-12) where the factor of $1 / 2$ is needed in order to retain the identity of photons in both spaces. In transitioning between spatial dimensions, total energy is conserved. See Equation (2-19). Another way to write Equation (2-19) is either Equation (2-21) or (2-22). The volume also changes from $n$-space to $(n-1)$-space, and vice versa, according to Equation (2-26), or Equation (2-27), depending on whether we wish to work with latent heat density or latent heat.

We considered the particular phase transition from $n=4$ to $(n-1)=3$. To give a specific example for how the generalized CC relation works, we assumed a specific value for transition temperature, as well as a particular value for latent heat. We then calculated particular values for the internal energy density, entropy density, and volume both before and after the phase transition. We found that if we assume that $T=T_{43}=T_{34}=3 \times 10^{27}$ Kelvin, and, furthermore, if we take $L$ to equal $1.8 \times 10^{94}$ Joules, then we have:

$$
u_{4}=1.15 \times 10^{125} \mathrm{~J} \cdot \mathrm{m}^{-4}, \quad s_{4}=4.81 \times 10^{97} \mathrm{~J} \cdot \mathrm{m}^{-4} \cdot \mathrm{K}^{-1}, \quad V_{4}=2.14 \times 10^{-31} \mathrm{~m}^{4},
$$

with

$$
u_{3}=6.13 \times 10^{94} \mathrm{~J} \cdot \mathrm{m}^{-3}, \quad s_{3}=2.72 \times 10^{67} \mathrm{~J} \cdot \mathrm{m}^{-3} \cdot \mathrm{K}^{-1}, \quad V_{3}=0.267 \mathrm{~m}^{3}
$$

The subscripts 3, 4 refer to the dimension of space where the quantity is defined. We have considered only black-body photon radiation in order to keep the discussion simple. We notice a tremendous decrease in entropy in transitioning from $n=4$ to $(n-1)=3$ space, as well as a dramatic change in volume. The volume $V_{4}$ is defined in 4-space whereas $V_{3}$ is a three-dimensional construct; as such they cannot readily be compared. Nevertheless, $V_{3}$ is a subspace of $V_{4}$ because compactification will curl up one of the space dimensions. We remark that the latent heat released was assumed substantial, and we believe that it is released in the residual $n=3$ space as we discount exotic scenarios such as parallel universes.

The 4-volume, $V_{4}$, can be calculated once the latent heat, $L$, is known and vice versa. We assume that the $V_{3}$ value is known since the cosmic scale parameter is determined by the temperature, and the temperature is specified. The $V_{3}$ value at transition temperature $T_{34}=T_{43}$ must be equal to $V_{3}=V_{0} a^{-3}$ where " $a$ " is the cosmic scale parameter, and $V_{0}$ is the present size of the observable universe. Since $a=T_{0} / T_{43}$ where $T_{0}=2.725 \mathrm{~K}$, and since the radius of the observable universe is, at present, $4.4 \times 10^{26}$ meters, we calculate for $V_{3}$ a 
value of $0.267 \mathrm{~m}^{3}$ at a temperature of $T_{43}=3 \times 10^{27} \mathrm{~K}$. The relation between $V_{4}$ and latent heat, $L$, is a linear relation with an increase in $L$ leading to an increase in $V_{4}$. See Equation (3-12), or what is equivalent, Equation (3-11). A graph for $V_{4} / V_{3}$ versus $L$, for various $L$ values, is illustrated in Figure 1 .

The numbers calculated above have a direct connection to a previous work by the author [11] on inflation. We treated inflation as an isothermal expansion process, within a greater Carnot heat engine cycle. We hypothesize in this paper that the beginning of the isothermal process may have started with a $n=4$ to $(n-1)=3$ phase transition. This would account for the tremendous amount of heat release, which is needed for the isothermal process, from points $a \rightarrow b$ in the cycle. While this is conjecture, the numbers are seen to have the right order of magnitude. In addition, when we focus on the inhomogeneity in temperature in WMAP and Planck maps, which is of the order of $\delta T / T= \pm 5 \times 10^{-5}$, we find that the temperature fluctuations can be produced from one spatial dimension to the next when transitioning between spaces. See, for example, Equation (4-12) and Equation (4-13). If both neighboring spaces allow for adiabatic expansion/contraction, then there will be a smooth carry-over of temperature inhomogeneity from one spatial dimension to the next. This seems to be a special feature of our generalized CC relation. The specific thermodynamic variables vary in a characteristic way with respect to a variation in temperature. See Equation (4-1), Equation (4-2). If we assume adiabatic expansion or adiabatic contraction in $n$-dimensional space, then we have the further relations, Equations (4-3)-(4-7).

Higher order spatial phase transitions can be considered, e.g. from $n=5$ to $(n-1)=4$, from $n=6$ to $(n-1)=5$, etc. We can apply the generalized CC relation, Equation (2-12), to these situations. If we multiply Equation (2-12) by negative one, left and right hand sides, we can also transition in reverse, from $(n-1)$ spatial dimensions to $n$-spatial dimensions. Now latent heat must be supplied for the process to happen, as entropy will increase as well as internal energy density.

If we decrease the number of spatial dimensions, then we can only transition from $n=3$ to $(n-1)=2$, and from $n=2$ to $(n-1)=1$. We notice that the internal energy density, specified by Equation (2-1), is infinite for $n=0$ as we are then dividing by $\Gamma(0)$, which is in the denominator and is zero. If $n=1$ is substituted in Equation (2-1), then the denominator is well defined, but we obtain a zero value in the numerator. Radiation energy cannot exist in a 1-dimensional space. Nevertheless, a transition from $n=2$ to $(n-1)=1$ is a possibility. As the dimension decreases, there is less latent heat released, and the energy densities decrease as well. The entropy also decreases, as more space allows for more disorder, and less space means less disorder.

Finally, we close this paper with the observation that space, in and of itself, must have energy. We know that space filled with radiation has energy. This is obvious from Equation (2-1) because any finite temperature above absolute zero will give us a finite energy density for $\mathrm{n}$ greater than one. The energy is trapped 
in the radiation itself, i.e. within the photons, within a given dimension. What we have shown in this work is that if one gives up space, by decreasing the dimension, one automatically releases latent heat. When one adds space, by increasing the spatial dimension, then one has to necessarily supply latent heat. Therefore, space itself must have energy content since transitioning between spaces supplies or costs energy. In other words, the latent heat supplied can be either positive or negative depending on the direction of the spatial transition. We can quantify the amount of energy released and taken in, when switching from one spatial dimension to another, with our generalized CC relation. This is the most spectacular result of this paper.

The author would like to thank Gonzaga University, and the Physics Department, in particular, for their support and encouragement. Special thanks goes to Dr. Eric Aver and Dr. Adam Fritsch for reading the manuscript, and offering advice. I also thank John Krehbiel for many insightful discussions. Any shortcomings, however, are entirely those of author.

\section{Conflicts of Interest}

The author declares no conflicts of interest regarding the publication of this paper.

\section{References}

[1] Wark, K. and Richards, D. (2001) Thermodynamics. 6th Edition, McGraw-Hill, Inc., New York, NY.

[2] Çengel, Y.A. and Boles, M.A. (2019) Thermodynamics-An Engineering Approach. McGraw-Hill Series in Mechanical Engineering. 9th Edition, McGraw-Hill, Inc., Boston, MA.

[3] Salzman, W.R. (2001) Clapeyron and Clausius-Clapeyron Equations. Chemical Thermodynamics. University of Arizona, Tucson.

[4] Krafcik, M. and Sánchez Velasco, E. (2014) Beyond Clausius-Clapeyron: Determining the Second Derivative of a first-Order Phase Transition Line. American Journal of Physics, 82, 301-305.

[5] Klein, O. (1926) Quantentheorie und fünfdimensionale Relativitätstheorie. Zeitschrift für Physik A, 37, 895-906.

[6] Jordan, P. (1948) Fünfdimensionale Kosmologie. Astronomische Nachrichten, 276, 193-208.

[7] Appelquist, T., Chodos, A. and Freund, P.G.O. (1987) Modern Kaluza-Klein Theories. Addison-Wesley, Menlo Park.

[8] Wesson, P.S. (1999) Space-Time-Matter, Modern Kaluza-Klein Theory. World Scientific, Singapore. https://doi.org/10.1142/3889

[9] Wesson, P.S. and Ponce de Leon, J. (1995) The Equation of Motion in Kaluza-Klein Cosmology and Its Implications for Astrophysics. Astronomy and Astrophysics, 294, 1-7.

[10] Castellani, L., et al. (1991) Supergravity and Superstrings. Vol. 2, Chapter V.11, World Scientific Publishing, Singapore.

[11] Pilot, C. (2017) Modeling Cosmic Expansion, and Possible Inflation, as a Thermodynamic Heat Engine. Zeitschrift für Naturforschung A (ZNA), 74, 153-163. 
[12] Kolb, E. and Turner, M. (1994) The Early Universe.

[13] Mather, J.C., et al. (1999) Calibrator Design for the COBE Far-Infrared Absolute Spectrophotometer (FIRAS). The Astrophysical Journal, 512, 511-520. https://doi.org/10.1086/306805

[14] Husdal, L. (2016) On Effective Degrees of Freedom in the Early Universe. arXiv:1609.04979v3.

[15] Gonzalez-Ayala, J., Cordero, R. and Angulo-Brown, F. (2016) Is the (3+1)-d Nature of the Universe a Thermodynamic Necessity? EPL (Europhysics Letters), 113, 40006 .

[16] Landsberg, P.T. and De Vos, A. (1989) The Stefan Boltzmann Constant in an N-Dimensional Space. Journal of Physics A: Mathematics and General, 22, 1073-1084. https://doi.org/10.1088/0305-4470/22/8/021

[17] Menon, V.J. and Agrawal, D.C. (1998) Comment on "The Stefan-Boltzmann Constant in N-Dimensional Space". Journal of Physics A: Mathematics and General, 31, 1109-1110. https://doi.org/10.1088/0305-4470/31/3/021

[18] Barrow, J.D. and Hawthorne, W.S. (1990) Equilibrium Matter Fields in the Early Universe. Monthly Notices of the Royal Astronomical Society, 243, 608-609.

[19] Gonzalez-Ayala, J., Perez-Oregon, J., Cordero, R. and Angulo-Brown, F. (2015) A Possible Cosmological Application of Some Thermodynamic Properties of the Black Body Radiation in N-Dimensional Euclidean Spaces. Entropy, 17, 4563-4581. https://doi.org/10.3390/e17074563

[20] Equation 5.19.4, NIST Digital Library of Mathematical Functions. https://dlmf.nist.gov/5.19\#iii

[21] Wang, X. (2005) Volumes of Generalized Unit Balls. Mathematics Magazine, 8, 390-395. https://doi.org/10.2307/30044198

[22] Frequently Asked Questions in Cosmology. Astro.ucla.edu.

[23] Lineweaver, C. and Davis, T.M. (2005) Misconceptions about the Big Bang. Scientific American, 292, 24-33. https://doi.org/10.1038/scientificamerican0305-36 